\title{
Intra-arterial computed tomography angiography with ultra-low volume of iodine contrast and stent implantation in transplant renal artery stenosis in terms of contrast-induced kidney injury - a preliminary report
}

\author{
Agata Szczurowska ${ }^{1}$, Mirosław Banasik², Jacek Kurcz' ${ }^{1}$, Marcin Miś1 , Katarzyna Nowańska², \\ Katarzyna Madziarska², Oktawia Mazanowska², Magdalena Krajewska², Jerzy Garcarek', Maciej Guziński
}

'Department of General Radiology, Interventional Radiology and Neuroradiology, Wroclaw Medical University, Wroclaw, Poland

²Department and Clinic of Nephrology and Transplantation Medicine, Wroclaw Medical University, Wroclaw, Poland

\section{Abstract}

Purpose: Traditional digital subtraction angiography is still regarded as the gold standard in the diagnostics of transplant renal artery stenosis (TRAS). However, this procedure requires a high volume of iodine contrast medium for optimal visualisation of the renal artery. The aim of this study was to analyse both the usefulness and the safety of intra-arterial computed tomography angiography (IA-CTA) with ultra-low-volume iodine contrast administration in the diagnostic and therapeutic management of TRAS in patients with impaired renal transplant function.

Material and methods: Thirty-three patients with a suspicion of TRAS based on Doppler-ultrasound and clinical setting underwent IA-CTA with ultra-low iodine contrast volume. A special, author-elaborated CTA protocol was used. The volume of 8-18 ml of diluted iodine contrast medium was administered through a catheter with the tip placed $2 \mathrm{~cm}$ below the aortic bifurcation.

Results: In six patients the CTA examinations revealed TRAS in three configurations: in the anastomosis, in the trunk (critical and high-grade), or in both sections. Stenoses were treated with primary stenting obtaining favourable anatomical outcome. No intervention-related complications were observed. No contrast-induced acute kidney injury was diagnosed in this study. Mean serum creatinine concentration was $2.93 \pm 0.89 \mathrm{mg} / \mathrm{dl}$ at the baseline and $2.89 \pm$ $1.73 \mathrm{mg} / \mathrm{dl}$ and $2.17 \pm 0.51 \mathrm{mg} / \mathrm{dl}$ after three and seven days from IA-CTA, respectively.

Conclusions: Intra-arterial CTA with ultra-low volume of iodine contrast seems to be a safe and reliable diagnostic tool to detect and assess TRAS in the aspect of stent implantation. Application of this imaging modality eliminates the need for a high volume of iodine contrast and thus does not adversely influence renal transplant function.

Key words: intra-arterial CTA, intra-arterial CTA with ultra-low volume of iodine contrast, transplant renal artery stenosis.

\section{Introduction}

Transplant renal artery stenosis (TRAS) is a potentially reversible complication of kidney transplantation that occurs in 1-23\% of graft recipients [1]. Symptoms include arterial hypertension, vascular murmur over the graft, and renal function impairment. Ultrasound with Doppler imaging that reveals high systolic peak velocity of blood flow in the transplant renal artery (TRA) remains the screening diagnostic measure for TRAS. Traditional

Correspondence address:

Agata Szczurowska, Department of General Radiology, Interventional Radiology and Neuroradiology, Wroclaw Medical University, Wroclaw, Poland, e-mail: agata.szczurowska@gmail.com

Authors' contribution:

A Study design · B Data collection · C Statistical analysis · D Data interpretation · E Manuscript preparation · F Literature search · G Funds collection

Supported by:

Wroclaw Medical University (grant SUB.C270.19.057) 
angiography is used in both the diagnostics and therapy for this disorder. During angiography balloon angioplasty with or without stent implantation may be performed. The angiography requires a high volume of iodine contrast to be administered into the TRA in order to visualise the vessel, asses the stenosis, and eventually to implant the stent. There is a risk of progression of chronic allograft injury in the graft recipients with impaired graft function through contrast-induced acute kidney injury (CI-AKI) or contrast-induced nephropathy (CIN). CI-AKI is diagnosed when the serum creatinine level increases by $0.5 \mathrm{mg} / \mathrm{dl}$ or $25 \%$ (in relation to the initial level) within three days from contrast administration [2]. CT angiography with intra-arterial ultra-low volume of iodine contrast administration (IA-CTA) is a relatively new method used in the diagnosis of TRAS and in the search for the best potential stent location in patients with advanced TRAS. IA-CTA enables excellent TRA visualisation with ultralow volume of contrast applied locally during minimally invasive procedure.

The aim of this study was to evaluate the potential usefulness and transplant safety of intra-arterial CT angiography with ultra-low-volume contrast administration in the management of TRAS with impaired graft function in patients in whom stent implantation was necessary.

\section{Material and methods}

Thirty-three graft recipients with suspicion of TRAS based on clinical setting (persistent hypertension, elevated serum creatinine, bruit over the graft) and elevated peak systolic velocity in TRA ( $>200 \mathrm{~cm} / \mathrm{s}$ ) on Dopplerultrasound examination were considered for examination with IA-CTA. Fifteen patients were disqualified due to: significant graft dysfunction demanding dialysis, other causes of graft function deterioration (high level of calcineurin inhibitors, rejection, infections), clinical symptoms improvement, or lack of consent for an invasive procedure [3]. Eighteen graft recipients were examined with IA-CTA. In 12 patients (67\%) IA-CTA revealed difficult anatomical conditions (including significant or multiple kinking) that made stenting impossible due to high risk of complications (for example complete occlusion of TRA). In six (33\%) (three men and three women) graft recipients examined with IA-CTA severe stenosis eligible for stent implantation was revealed. The six graft recipients were treated with TRA stenting during the same procedure, preceded by thorough assessment of the TRAS in CT image reconstructions performed by the operator. Informed consent was obtained in each case of IA-CTA and stent implantation.

The IA-CTA was performed with an author-elaborated protocol using 8-18 $\mathrm{ml}$ of diluted $30 \%$ iodine contrast (iodixanol 320) administered at the speed of $4-5 \mathrm{ml} / \mathrm{s}$ through catheter placed about $2 \mathrm{~cm}$ below the aortic bifurcation. The catheter was inserted using either an ipsilateral or contralateral inguinal arterial approach with the use of low-profile equipment (4F sheaths and catheters) in a cath lab located next door to the CT examination room. CTA examinations were performed with 64-slice LightSpeed CT (GE Healthcare) and analysed using an AW 4.4 (GE Healthcare) diagnostic station either manually or with Vessel Analysis software. In order to assess the length and severity of TRA narrowing 3D MIP (Maximum Intensity Projection) and CPR (Curved Planar Reconstruction) were made. After the analysis of stenosis, the stent implantation was performed. In four patients additional predilatation was done. In five patients correct patency of TRA was obtained, while in one TRA remained patent at ultra-low level. Serum creatinine level was measured at baseline and on each of the seven following days. EGFR was calculated using MDRD (modification of diet in renal disease) formula: GFR $=175 \times$ standardised Scr -1.154 $\times$ age $-0.203 \times 1.212$ [if black] $\times 0.742$ [if female], where GFR is expressed as $\mathrm{ml} / \mathrm{min} / 1.73 \mathrm{~m}^{2}$ of body surface area and Scr is expressed in $\mathrm{mg} / \mathrm{dl}$ [4].

\section{Results}

The average age of the patients was equal to $54.3 \pm 10.5$ years, CTA was performed 0.5 to 75.5 months after transplantation, volume of the contrast given during ia-CTA was $8-18 \mathrm{ml}$, and mean serum creatinine concentration at baseline was $2.93 \pm 0.89 \mathrm{mg} / \mathrm{dl}$.

IA-CTA revealed critical stenosis in the trunk and the anastomosis in three patients, critical stenosis in the trunk in one, $70 \%$ stenosis of the trunk in one, critical stenosis in the anastomosis in one, and additional kinking of TRA in one case (Table 1).

Stent administration resulted in good anatomical outcome. No complications of either arterial puncture or catheter insertion were observed.

No long-term graft dysfunction was observed in the group. Mean serum creatinine measured three days after contrast administration was $2.89 \pm 1.73 \mathrm{mg} / \mathrm{dl}$. One patient presented a creatinine level elevation of $1.9 \mathrm{mg} / \mathrm{dl}$, which decreased below the initial value within the four following days. In one patient elevation of $0.03 \mathrm{mg} / \mathrm{dl}$ was observed. In one case the creatinine remained at the same level. In three patients a decrease in serum creatinine level occurred within three days from the procedure. In prolonged measurement mean creatinine level after seven days from CTA was lower and equalled $2.17 \pm 0.51 \mathrm{mg} / \mathrm{dl}$. The decrease of serum creatinine level between baseline and day 7 was $0.2-1.64 \mathrm{mg} / \mathrm{dl}$ (mean $0.76 \pm 0.54 \mathrm{mg} / \mathrm{dl}$ ). Figure 1 presents the serum creatinine level change trends on each of the seven following days after transplantation for all six patients. Mean eGFR (estimated glomerular filtration rate) was $6.3 \mathrm{ml} / \mathrm{min} / 1.73 \mathrm{~m}^{2}$ higher than at the baseline.

\section{Discussion}

Vascular complications of renal transplantation, including TRAS, can result in graft loss. An effective screening 
Table 1. The data of patients, denoted as A-F, including their age, sex ( $\mathrm{M}$ - male, $\mathrm{F}$ - female), time from transplantation, total volume of contrast administered, volume of contrast injected at IA-CTA, type of stenosis, and serum creatinine level ( $\mathrm{SCr}$ ) at baseline

\begin{tabular}{|l|c|c|c|c|c|c|c|}
\hline & Age & Sex & $\begin{array}{c}\text { Time after } \\
\text { transplantation } \\
\text { (months) }\end{array}$ & $\begin{array}{c}\text { Volume of contrast } \\
\text { in IA-CTA (ml) }\end{array}$ & $\begin{array}{c}\text { Total volume of } \\
\text { contrast (IA-CTA + stent } \\
\text { implantation) (ml) }\end{array}$ & $\begin{array}{c}\text { Type and severity } \\
\text { of stenosis }\end{array}$ & $\begin{array}{c}\text { SCr at the } \\
\text { baseline } \\
\text { (mg/dl) }\end{array}$ \\
\hline A & 63 & M & 5 & 8 & 100 & Trunk + anastomosis; critical & 3.5 \\
\hline B & 61 & F & 7 & 8 & 100 & Trunk + kinking; critical & 2.5 \\
\hline C & 64 & M & 75.5 & 15 & 15 & Anastomosis; critical & 1.9 \\
\hline D & 54 & F & 0.5 & 18 & 168 & Trunk + anastomosis; critical & 4.4 \\
\hline E & 46 & M & 3.5 & 8 & 80 & Trunk; $70 \%$ & 2.8 \\
\hline F & 38 & M & 5.5 & 8 & 100 & Trunk + anastomosis; critical & 2.5 \\
\hline
\end{tabular}
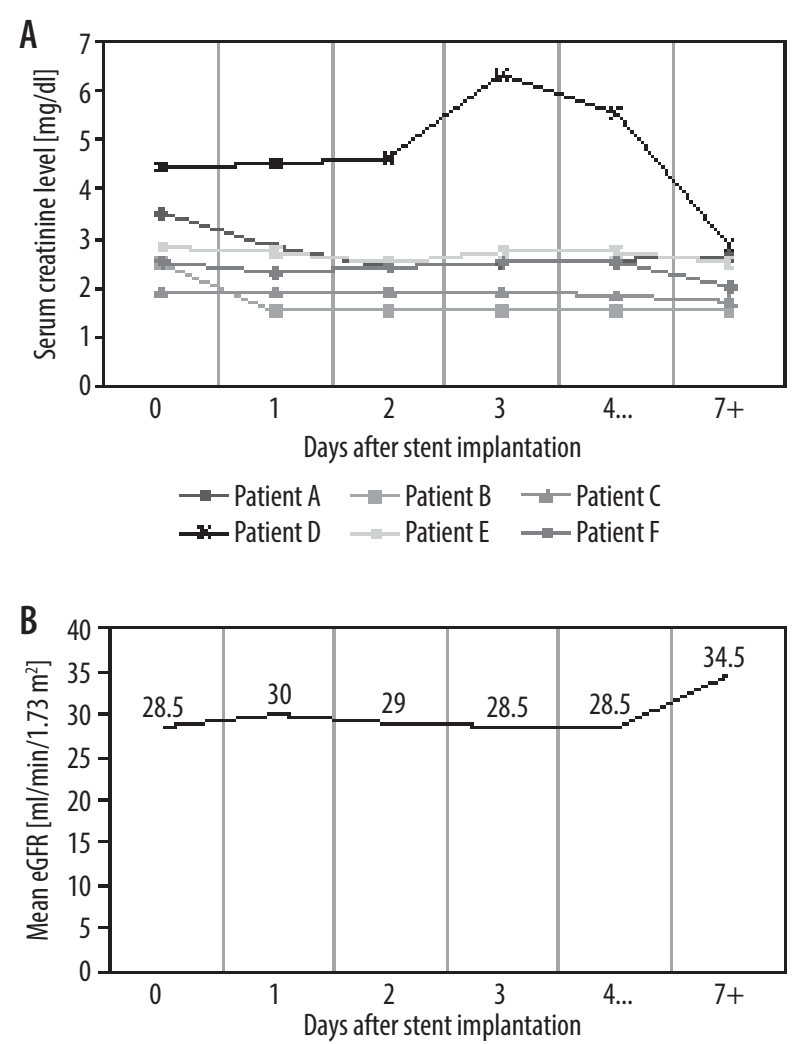

Figure 1. The serum creatinine level alterations $(A)$ and mean estimated glomerular filtration rate (eGFR) value (B) after stent implantation in over 7-day observation

test and possibility of fast treatment introduction are essential to maintain correct graft function. Several studies have been conducted in search of a sensitive and specific diagnostic measure for TRAS detection with as little invasion as possible.

Etemadi et al. [5] proved that high levels of $\mathrm{CaPO}_{4}$ product and LDL cholesterol are significantly correlated with the risk of TRAS one year after renal transplantation, which can be used in defining a risk group for TRAS among graft recipients. Doppler ultrasound imaging remains a safe and accurate tool for TRAS diagnosis during systematic follow-up after kidney transplantation [6]. All patients in our study presented abnormalities in Doppler ultrasound examination of TRA after kidney trans-

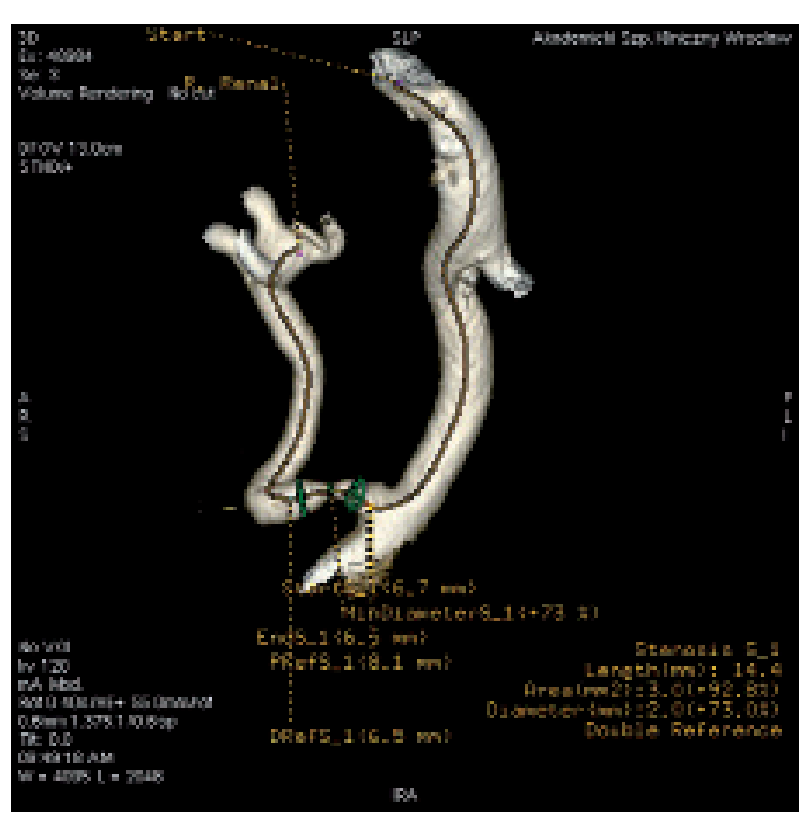

Figure 2. Intra-arterial computed tomography angiography with ultra-low volume of iodine contrast - a 3D reconstruction of the transplant renal artery with a detailed image of the stenosis

plantation. Contrast-enhanced ultrasound [7] as well as magnetic resonance angiography (MRA) [8] are accurate and relatively safe methods for further and more detailed assessment of TRAS; however, these methods are less available than CTA. Bashir et al. presented an alternative, non-nephrotoxic MRA with the use of ferumoxytol as contrast agent [9], which could become an optimal diagnostic measure for TRAS in the future. Regular computed tomography angiography enables detailed images of TRA abnormalities to be obtained. Nevertheless, it requires administration of a high volume of iodine contrast, which carries a risk of graft failure in patients with already impaired graft function. Several studies proved that ultra-low-volume iodine contrast administered intra-arterially during CTA ensures optimal technical feasibility of this imaging method $[10,11]$. Moreover, intra-arterial puncture can be used for percutaneous intraluminal angioplasty and or without stent implantation in one step with CTA on condition that 
there is a short distance between the cath lab and CT room [10], as it was in the cases presented in this study.

According to "European Society of Urogenital Radiology guidelines on contrast media" renal function should be measured within 48-72 hours after contrast application [2]. In this study renal graft function parameters were controlled for a longer period (minimum seven days) to check both the effect of applied treatment as well as the reaction to iodine contrast administered during IA-CTA and stent implantation. In all cases a significant decrease in serum creatinine level at the end point was observed. In addition to minimising the amount of contrast, benefits from stent implantation outweighed the potential risk of graft failure due to contrast administration. Therefore, it is reasonable to use ultra-low-volume IA-CTA, particularly while applying treatment designed to improve the graft function. Effectiveness of TRA stenting in TRAS reaches $80 \%$ [3] and enables graft function to be maintined for many years [12] if applied punctually.

This study had several limitations. No additional laboratory factors connected with TRAS were measured. The group of patients examined was small, but it should be noted that TRAS requiring stent implantation is rare in the general population. We did not take into account any control group because the number of the patients under examination was small and the purpose of the study was to present the potential of the relatively new method of imaging of severe TRAS. The use of CT scanners with 256 and more slices may potentially improve IA-CTA examinations in terms of volume of contrast medium and additional features (including graft perfusion).

\section{Conclusions}

Intra-arterial CTA using an ultra-low volume of iodine contrast seems to be a safe diagnostic tool to asses TRAS in need of stent implantation.

Application of IA-CTA with an ultra-low volume of iodine contrast may minimise the overall amount of contrast that patients receive in the management of TRAS.

\section{Conflict of interest}

The authors report no conflict of interest.

\section{References}

1. Fervenza FC, Lafayette RA, Alfrey EJ, Petersen J. Renal artery stenosis in kidney transplants. Am J Kidney Dis 1998; 31: 142-148.

2. European Society of Urogenital Radiology guidelines on contrast media, version 9.0. Available at: http://www.esur-cm.org/index.php/en/.

3. Banasik M, Garcarek J, Kurcz J, Klinger M. Transplant renal artery stenosis as the cause of arterial hypertension. Forum Nefrol 2012; 5: 30-36.

4. Levey AS, Stevens LA, Schmid CH, et al. A new equation to estimate glomerular filtration rate. Ann Intern Med 2009; 150: 604-612.

5. Etemadi J, Rahbar K, Haghighi AN, et al. Renal artery stenosis in kidney transplants: assessment of the risk factors. Vasc Health Risk Manag 2011; 7: 503-507.

6. Granata A, Clementi S, Londrino F, et al. Renal transplant vascular complications: the role of Doppler ultrasound. J Ultrasound 2015; 18: 101-107.

7. Grzelak P, Kurnatowska I, Nowicki M, et al. Detection of transplant renal artery stenosis in the early postoperative period with analysis of parenchymal perfusion with ultrasound contrast agent. Ann Transplant 2013; 18: 187-194.
8. Fananapazir G, Bashir MR, Corwin MT, Lamba R, Vu CT, Troppmann C. Comparison of ferumoxytol-enhanced MRA with conventional angiography for assessment of severity of transplant renal artery stenosis. J Magn Reson Imaging 2017; 45: 779-785.

9. Bashir MR, Jaffe TA, Brennan TV, Patel UD, Ellis MJ. Renal transplant imaging using magnetic resonance angiography with a nonnephrotoxic contrast agent. Transplant J 2013; 96: 91-96.

10. Garcarek J, Kurcz J, Guziński M, Banasik M, Miś M, Gołębiowski T. Intraarterial CT angiography using ultra low volume of iodine contrast - own experiences. Polish J Radiol 2015; 80: 344-349.

11. Althoff CE, Günther RW, Hamm B, Rief M. Intra-arterial ultra low iodine CT angiography of renal transplant arteries. Cardiovasc Intervent Radiol 2014; 37: 1062-1067.

12. Marini M, Fernandez-Rivera C, Cao I, et al. Treatment of transplant renal artery stenosis by percutaneous transluminal angioplasty and/ or stenting: study in 63 patients in a single institution. Transplant Proc 2011; 43: 2205-2207. 